\title{
Delineamento de um Agente para detectar a Auto-Eficácia do Aluno no Ensino-Aprendizagem via Web
}

Francine Bica*

Regina Verdin**

Rosa Maria Vicari***

\section{Resumo}

Este artigo apresenta o delineamento de um agente com o objetivo de detectar a autoeficácia do aluno no ensino-aprendizagem via Web. O delineamento emprega o uso do modelo Fuzzy para capturar a auto-eficácia do aluno e apresentar o feedback através de um agente animado que terá a função de apresentar o comportamento adequado. Os resultados experimentais realizados no contexto de ensino de Biologia mostram o uso do sistema a princípio sem o uso do agente. Eficácia

Palavras-Chaves: Educação a Distância, Sistema Multiagentes, Lógica Fuzzy, Auto-

\section{Abstract}

This article presents the design of an agent with the objective to detect the autoefficacy of the student in the teach-learning over the Web. The design prescribes the use of the Fuzzy model to capture the auto-efficacy of the student and presents a feedback through an agent who will have the function to present the adequate behaviour. The experimental results carried through in the context of education of Biology shows the use of the system without the use of the agent.

Keywords: Distance Education, Multiagent System, Fuzzy Logic, Self-Efficacy

\section{Introdução}

A utilização da tecnologia na educação on-line tem exigido reflexões sobre como podemos promover com maior eficácia o ensino-aprendizagem nos ambientes virtuais de ensino.Um dos fatores que falta ao ensino presencial, e que se detecta a sua ausência também no ensino virtual, seriam as habilidades de independência e iniciativa, as quais proporcionam ao aluno auto-regular-se na sua motivação (Peters, 2001). A auto-regulação da motivação está relacionada com as crenças que o aluno tem sobre a sua Auto-Eficácia (Bandura 1997), ou competência, a qual precisa ser considerada para o delineamento de ambientes de aprendizagem virtuais.

Sob a perspectiva do aluno, a Auto-Eficácia é uma crença funcional positiva sobre a competência que ele tem de si próprio em controlar o seu processo de aprendizagem usando as tecnologias educativas (Costa, 2002). Num âmbito geral, as crenças que as pessoas têm a respeito de sua eficácia acabam influenciando suas escolhas, suas aspirações, e a quantidade de esforço que elas mobilizam para executar uma tarefa, assim como a persistência diante das dificuldades (Bandura, 1997). As crenças de Auto-Eficácia determinam parcialmente as várias funções e subfunções do sistema auto-regulatório, e do processamento cognitivo, dos diferentes aspectos do desempenho, assim como dos resultados decorrentes deles. O senso de Auto-Eficácia é representado por crenças proposicionais (verbais) e estão imbuídas numa rede relacional e funcional com outros fatores cognitivos e afetivos no gerenciamento da realidade (Bandura, 1997).

\footnotetext{
* Aluna de Doutorado PPGC/UFRGS, Mestre em Informática, bolsista CNPq/UFRGS, francine@inf.ufrgs.br

** Aluno de Doutorado PGIE/UFRGS, Mestre em Psicologia, bolsista CNPq/UFRGS, rverdin@inf.ufrgs.br

**** Professora do Instituto de Informática, Pós Doutorada em Informática, rosa@inf.ufrgs.br, www.inf.ufrgs.br/ rosa
} 
Sob uma perspectiva computacional, os avanços tecnológicos mais recentes no campo dos ambientes de aprendizagem considerados inteligentes, têm proposto o uso de arquiteturas baseadas em sociedades de agentes. Esta abordagem tem mostrado um potencial bastante adequado ao desenvolvimento de ambientes de aprendizagem (Jaques 2003, Silveira 2002), devido ao fato da natureza do problema de ensino-aprendizagem ser mais facilmente resolvido de forma cooperativa, suportando um desenvolvimento de sistemas de forma mais robusta, mais rápida e com menores custos, tornando-os mais atrativos, do ponto de vista de seu aproveitamento real, não ficando restrito a um protótipo.

Agente é um conceito que define entidades inteligentes que podem apresentar, entre outras, as seguintes características (Wooldridge 2000, Lesser 2003): perceber dinamicamente as condições do ambiente e se adaptar a novas situações; tomar decisões para afetar condições do ambiente, exibindo comportamento direcionado a solução dos seus objetivos; interpretar percepções, resolver problemas, extrair inferências e determinar ações; exibir habilidade social e cooperar com outros agentes e com o usuário.

Este artigo apresenta o delineamento de um agente, o qual, dentro do ambiente de aprendizagem na Web, denominado InteliWeb, desempenhará o papel de gerenciador do modelo do aluno levando em consideração, principalmente, as crenças de Auto-Eficácia do mesmo. Estas crenças estão sendo dimensionadas através da aplicação piloto do ambiente InteliWeb na disciplina denominada Anatomia Vegetal do curso de Biociências da Universidade do Rio Grande do Sul. Participaram do experimento pesquisadores dos Departamentos de Informática, Informática na Educação e Biociências da Universidade Federal do Rio Grande do Sul.

Este trabalho está divido nas seguintes partes: o item 2 apresenta o modelo de Autoeficácia e trabalhos relacionados, no item 3 é apresentado o delineamento do agente gerenciador modelo do aluno, responsável por gerenciar o modelo do aluno, levando em consideração, principalmente, as crenças de Auto-Eficácia, no item 4 o ambiente Inteliweb e os procedimentos de avaliação deste são apresentados e no item 5 são abordados considerações finais e trabalhos futuros.

\section{A Auto-Eficácia no Modelo do aluno}

A representação do aluno no sistema (modelo do aluno), é uma das partes mais complexas e, ao mesmo tempo, frágeis de um ambiente de aprendizagem, devido a fatos como: o pouco conhecimento que se tem sobre os processos de aprendizagem de um aluno, a imprecisão e subjetividade dos fatores emotivos e motivacionais envolvidos em ambientes de ensino e aprendizagem e o problema da representação do conhecimento pedagógico.

O modelo do aluno, no contexto de Tutores Inteligentes, representa o conhecimento e as habilidades cognitivas do aluno em um dado momento, sendo assim, a modelagem do aluno requer a interdisciplinaridade entre Educação, Psicologia da Aprendizagem e Ciências Cognitivas.

Existem variados modelos de alunos na literatura, um dos focos da pesquisa de modelos de aluno está permitindo que um usuário negocie com o seu modelo (Kay, 2001), outra linha de pesquisa foca a questão de como dar suporte a grupos de pessoas, de forma a permitir que estas se tornem times de trabalho (Johnson et al., 1997).

Tedesco (2001) propõem criar e manter modelos de grupo em sistemas colaborativos. Existem propostas de modelos de alunos de caráter gerencial, como propõem diversos órgãos internacionais (PAPI, 2004). Outra área de estudo é a incorporação de emoções ao modelo do aluno, como nos trabalhos de Bercht (2001) e Soldato (1995).

Neste trabalho, o modelo de aluno gerenciado pelo agente Gerenciador do Modelo do Aluno espera proporcionar ao aluno uma mediação pedagógica, no qual será realizada a tentativa de promoção, principalmente, da sua eficácia, e conseqüentemente da motivação.

Esta orientação almeja que o aluno não se perca na tarefa, não se sinta sozinho ou não se mobilize negativamente para desempenhar sua aprendizagem. Para isso, esta 
pesquisa busca um modelo de aluno que possa contemplar a dinamicidade necessária e que suporte a individualidade do aluno através da modelagem das crenças da auto-eficácia.

A utilização da característica individual da auto-eficácia é importante para a modelagem cognitiva, na medida que, conforme Bandura, é ela que permeia o controle e regulação pessoal do aluno na sua motivação durante a construção do seu conhecimento no processo de aprendizagem. Nesse sentido, quanto mais um sistema computacional (tutor) tiver a capacidade de capturar a auto-eficácia do aluno, tanto mais este poderá fomentar no aluno a crença na sua própria capacidade de exercer controle sobre a sua aprendizagem.

Bandura (1997) desenvolveu a teoria da Auto-Eficácia. Esta teoria defende que todos os processos de mudança psicológica e comportamental se operam a partir de alterações no sentido de domínio e Auto-Eficácia. Este autor define como Auto-Eficácia "a crença do indivíduo sobre as suas capacidades de exercer controle sobre acontecimentos que afetam a sua vida", "e a crença nas suas capacidades para mobilizar motivação, recursos cognitivos e implementar ações que lhe permitam exercer controle sobre tarefas exigidas".

Desta forma, a Auto-Eficácia diz respeito, não só as capacidades que um indivíduo possui para realizar determinada tarefa com sucesso, mas sim ao julgamento que ele faz sobre essas mesmas capacidades (Maddux, 1995). As crenças acerca destas capacidades e recursos pessoais constituem em um produto da interação entre diversos fatores, como experiências anteriores de sucesso ou fracasso.

No contexto acadêmico Bzuneck (2004) declara que um aluno motiva-se se envolver nas atividades de aprendizagem caso acredite que, com os seus conhecimentos, talentos e habilidades, poderá adquirir novos conhecimentos, dominar um conteúdo, melhorar suas habilidades, entre outros. Assim, esse aluno selecionará atividades e estratégias de ação que, segundo prevê, poderão ser executadas por ele e abandonará outros objetivos ou cursos de ação que não lhe representem incentivo, porque sabe que não os poderá implementar.

A Auto-Eficácia pode ser representada como um esquema ou modelo de funcionamento mental. O modelo de Auto-Eficácia proposto por Bandura possui como principais elementos: objetivos, padrão pessoal, processo avaliativo e feedback. A Figura 1 apresenta esse modelo e as relações entre seus elementos.

Seguindo os pressupostos de Bandura (1997), as crenças de Auto-Eficácia influenciam as escolhas dos objetivos por parte do aluno. No modelo computacional proposto, estes objetivos, chamados de objetivos de aprendizagem podem ser extrínsecos ou intrínsecos.

Como objetivo intrínseco tem-se o domínio do conhecimento e como objetivo extrínseco o desempenho. 


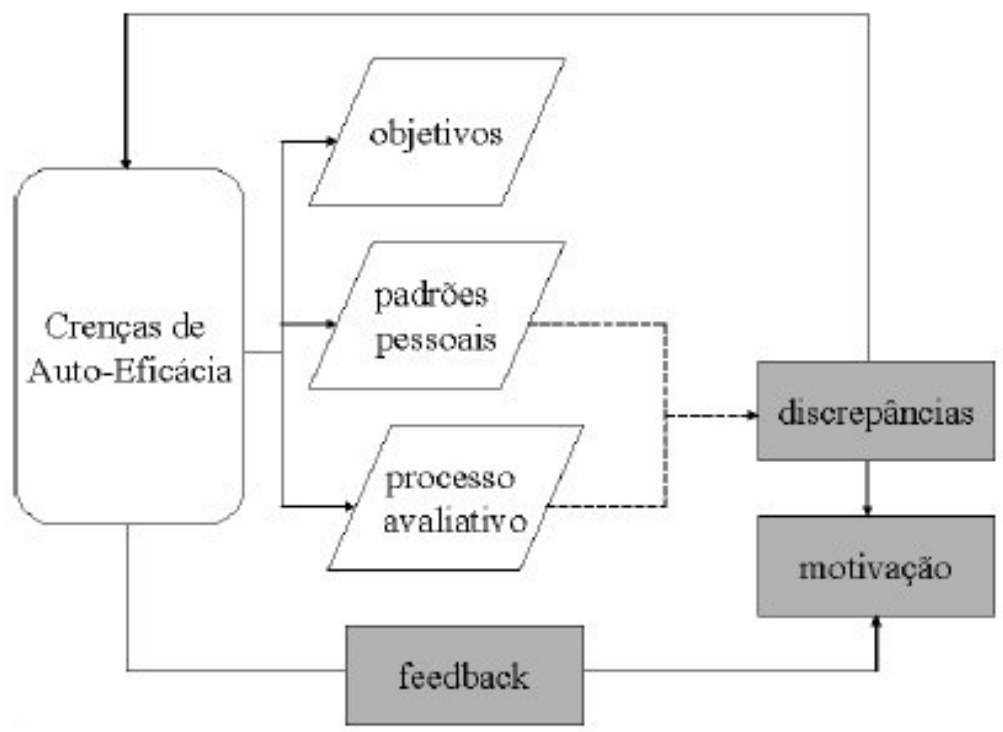

Figura 1 - Modelo de Auto-Eficácia

Cerdeira (1995) e Bandura (1997) sustentam que quando se permite aos indivíduos as escolhas de suas metas, estes assumem para com elas um compromisso, passando deste modo a considerarem-se responsáveis pelos progressos relativos a sua persecução, incrementando assim os sub-processos da auto-avaliação e, por isso também, o nível de desempenho e as expectativas de Auto-Eficácia.

Quando o aluno seleciona um objetivo ele cria um padrão pessoal de si mesmo, ou seja, o que ele espera de si mesmo na realização de uma atividade. Contudo, no decorrer das suas ações, pode acontecer do aluno se desviar do objetivo selecionado e através de um processo avaliativo, seja, por exemplo, por exercícios, provas ou tempo despendido na sessão de estudo, podem ocorrer discrepâncias. Estas discrepâncias percebidas pelo aluno afetam diretamente a sua motivação e as crenças de Auto-Eficácia.

Um sistema de feedback deve ser acionado quando ocorrem tais discrepâncias. Segundo Lock (1990) e Bandura (1997) inicialmente os objetivos por si só servem de impulsionadores da ação aumentando a motivação do aluno, mas se não houver um sistema de feedback que regule e controle as ações, os objetivos podem acabar perdendo a sua força.

Nesse contexto o modelo do aluno proposto neste trabalho engloba as crenças da autoeficácia, o objetivo de aprendizagem, o padrão pessoal, o esforço e a persistência. $\mathrm{O}$ grau de esforço pode ser entendido como a intensidade na realização das atividades para atingir um alvo e é mapeado como o tempo no qual o aluno permaneceu fazendo uma tarefa. Já a persistência pode ser entendida como a constância em uma atividade (Soldato, 1995) (Arison, et. al, 1990).

A auto-Eficácia vem sendo investigada na aprendizagem a distância e no uso de computadores. Os resultados das pesquisas de Levine (1998) mostram que os alunos que têm alta Auto-Eficácia demonstram atitudes positivas em relação ao uso de computadores para sua aprendizagem. Joo et al (2000) investigou a influencia da Auto-Eficácia na autoregulação dos alunos em sua aprendizagem, no desempenho acadêmico, e Internet e no desempenho na instrução via web, concluindo que a Auto-Eficácia é uma variável importante que determina o sucesso na aprendizagem.

Nos resultados dos estudos de (O'Malley et al., 1999) demonstram que os alunos geralmente não percebem a educação a distância tão eficaz quanto os métodos tradicionais.

No entanto, foi constatado que aprendizagem a distância tem alguma vantagem tais como trabalhar com tempo determinado pelo aluno. 


\section{Delineamento do Agente Gerenciador do Modelo do Aluno}

$\mathrm{O}$ agente gerenciador do modelo do aluno (AGMA) tem a capacidade de perceber e monitorar a Auto-Eficácia do aluno e prover o modelo do aluno com esta variável. Este agente promoverá, quando necessário, feedbacks ao aluno através do PAT (agente pedagógico animado) (Jaques 2003), durante a sua sessão de estudo, com o objetivo de promover a Auto-Eficácia.

A base de dados armazena o log dos passos do aluno durante a sessão de estudo, além de dados cadastrais do curso e outros dados utilizados pelo InteliWeb. É através desse $\log$ que o agente captura as variáveis para realizar o processo de inferência da AutoEficácia, assim como atualizações no modelo do aluno.

O modelo de Auto-Eficácia incorporado ao AGMA foi baseado em Bandura (1997), contudo agregou-se a hipótese da utilização da persistência como uma das variáveis para a inferência da Auto-Eficácia, além do esforço e padrão pessoais sugeridos no modelo de Bandura.

A Figura 2 apresenta as quatro atividades do AGMA em relação a Auto-Eficácia, as quais são: (1) processo avaliativo, (2) análise das discrepâncias, (3) inferência das crenças da Auto-Eficácia e (4) seleção do feedback. Acompanhando a Figura 2, o aluno inicia a sessão de estudo, na qual ocorre a sua identificação, uma estimativa de tempo em que permanecerá na sessão e a seleção do seu objetivo de aprendizagem. Com base nestes dados o agente recupera ou gera um modelo de aluno, se este não existir. Se o modelo do aluno já existir o PAT pode ser acionado com uma mensagem coerente com a Auto-Eficácia inferida previamente.

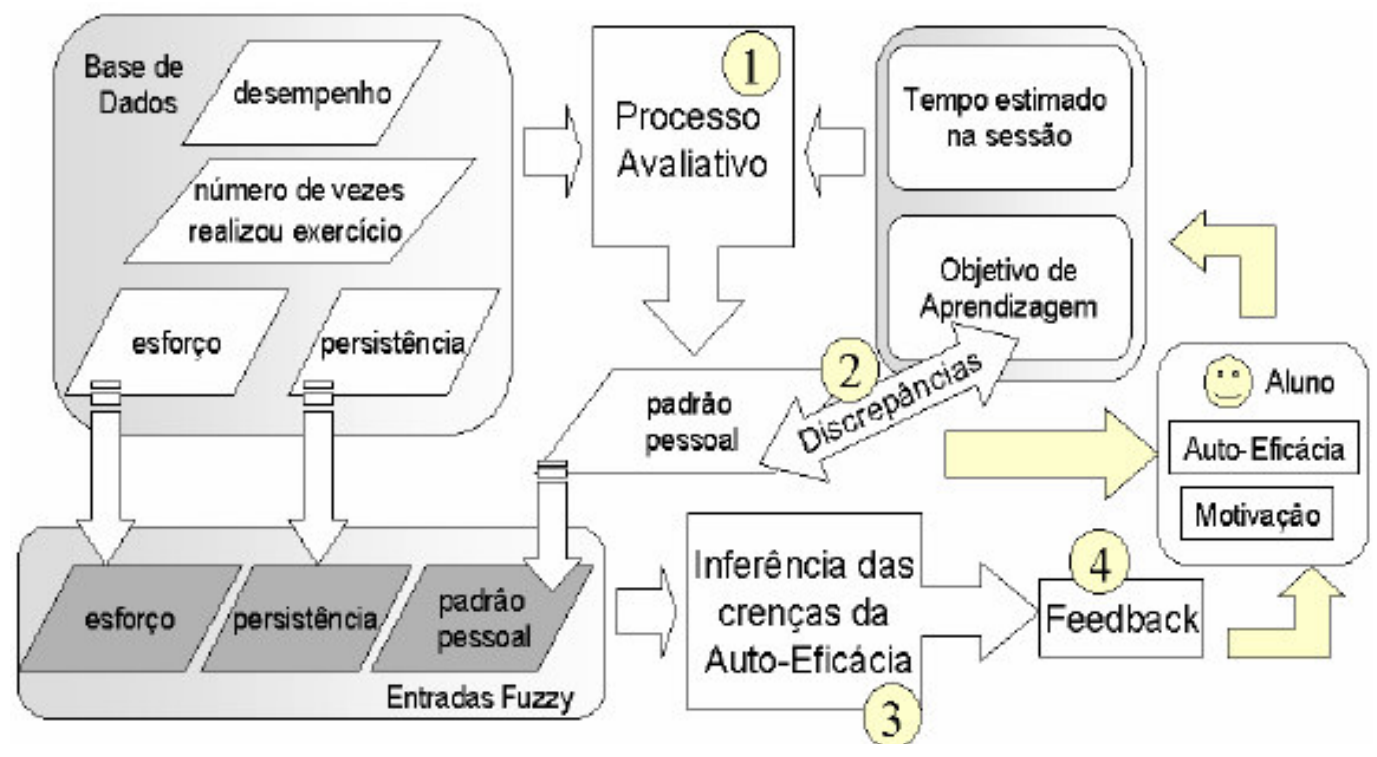

Figura 2 - Atividades do AGMA referentes a Auto-Eficácia

A navegação do aluno no material didático é livre e gera um log que é constantemente analisado pelo agente. É através desse log que ele recupera informações a respeito do esforço e persistência, além de desempenho nos exercícios, número de vezes que realizou os exercícios e tempo de permanência na sessão.

$\mathrm{Na}$ atividade processo avaliativo o agente infere o padrão pessoal a respeito do aluno, ou seja, se as ações do aluno, registradas no $\log$, foram coerentes com o seu objetivo de aprendizagem selecionado no início da sessão. Se houverem discrepâncias entre o padrão pessoal e o objetivo de aprendizagem, o agente revisa as crenças da Auto-Eficácia sobre o aluno. Após o processo avaliativo o modelo do aluno é novamente atualizado. A inferência 
das crenças da Auto-Eficácia é realizada através de um sistema Fuzzy composto pelos conjuntos esforço, pertinência e padrão pessoal, ilustrados na Figura 3.

Através da ajuda de um especialista instrucional (professor e autor do conteúdo da disciplina utilizada no InteliWeb), e da psicóloga do grupo foram especificadas as funções de pertinência para cada um dos conjuntos, as quais incluem as funções: trapezoidal, triangular, crescente e decrescente.

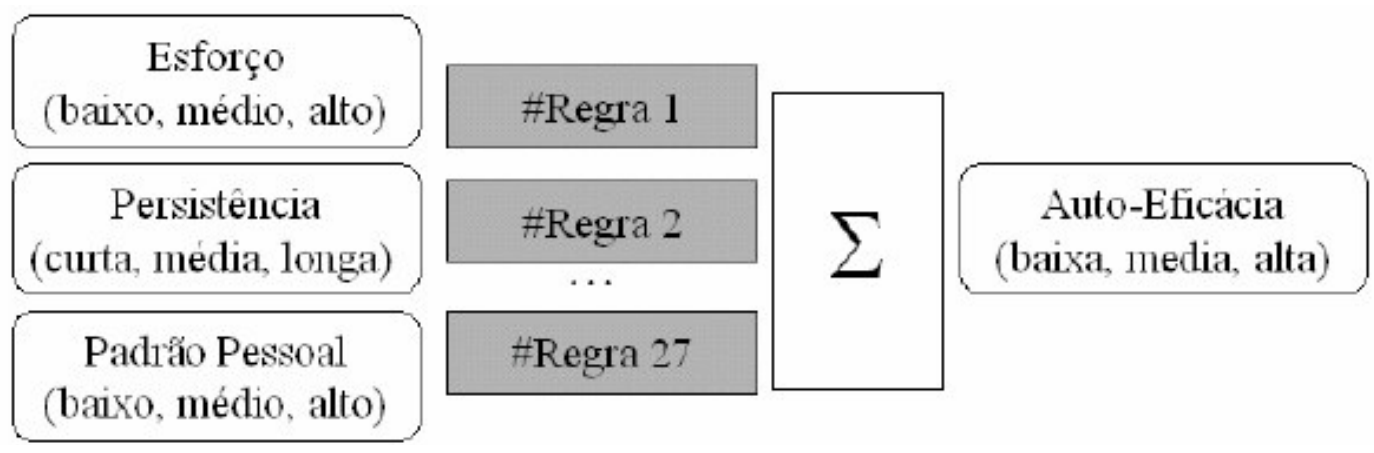

Figura 3 - Sistema Fuzzy da Auto-Eficácia

Exemplos de regras:

\#Regra 5 Se Esforço $=$ baixo $^{\wedge}$ Persistência $=$ media $^{\wedge}$ Padrão pessoal $=$ médio $\square$ Auto-Eficácia $=$

media

\#Regra 7 Se Esforço $=$ baixo $^{\wedge}$ Persistência $=$ longa ${ }^{\wedge}$ Padrão pessoal $=$ baixo $\square$ Auto-Eficácia $=$ baixa \#Regra 11 Se Esforço $=$ médio $^{\wedge}$ Persistência $=\operatorname{curta}^{\wedge}$ Padrão pessoal $=$ médio $\square$ Auto-Eficácia $=$

média

\#Regra 12 Se Esforço $=$ médio $^{\wedge}$ Persistência $=\operatorname{curta}^{\wedge}$ Padrão pessoal $=$ alto $\square$ Auto-Eficácia $=$ baixa \#Regra 18 Se Esforço $=$ médio $^{\wedge}$ Persistência $=$ longa ${ }^{\wedge}$ Padrão pessoal $=$ alto $\square$ Auto-Eficácia $=$ alta

O AGMA, a partir das funções de pertinência do conjunto esforço transforma o grau de esforço capturado do log em um valor fuzzy. O mesmo ocorre com a persistência e com o padrão pessoal. O operador composicional de inferência utilizado é o Max-min E, finalmente, na defuzzyficação o valor da variável lingüística de saída será traduzido para um valor discreto numérico, com o objetivo de obter-se o melhor valor que represente a crença da auto-eficácia. Entre os métodos de desfuzzyficação foi selecionado o método Centro da Área.

A auto-eficácia está indiretamente relacionada à motivação (Bandura, 1997). Na revisão das crenças de auto-eficácia o agente ativa um feedback, para proporcionar que o aluno controle e regule as suas ações, e assim diminuir a discrepância entre o estado atual (resultado do processo da avaliativo) e o desejado (objetivo de aprendizagem). Procura-se promover, assim, um aumento na Auto-Eficácia e, conseqüentemente, na motivação do aluno.

Para prover o feedback selecionado pelo AGMA o agente pedagógico animado selecionado foi a PAT, uma vez que, agentes pedagógicos animados podem aumentar a motivação do estudante, e possivelmente a sua atenção. O PAT apresenta comportamentos verbais e físicos afetivos e para cada tipo de comportamento (por exemplo: comportamento encorajamento), existem várias animações diferentes que podem ser exibidas. A Figura 4 ilustra três dos comportamentos selecionados. O comportamento (a) e (c) ilustram comportamentos físicos diferentes para uma "Nova Habilidade" e o (b) é um dos comportamentos verbais "Aumentar o esforço do estudante". 


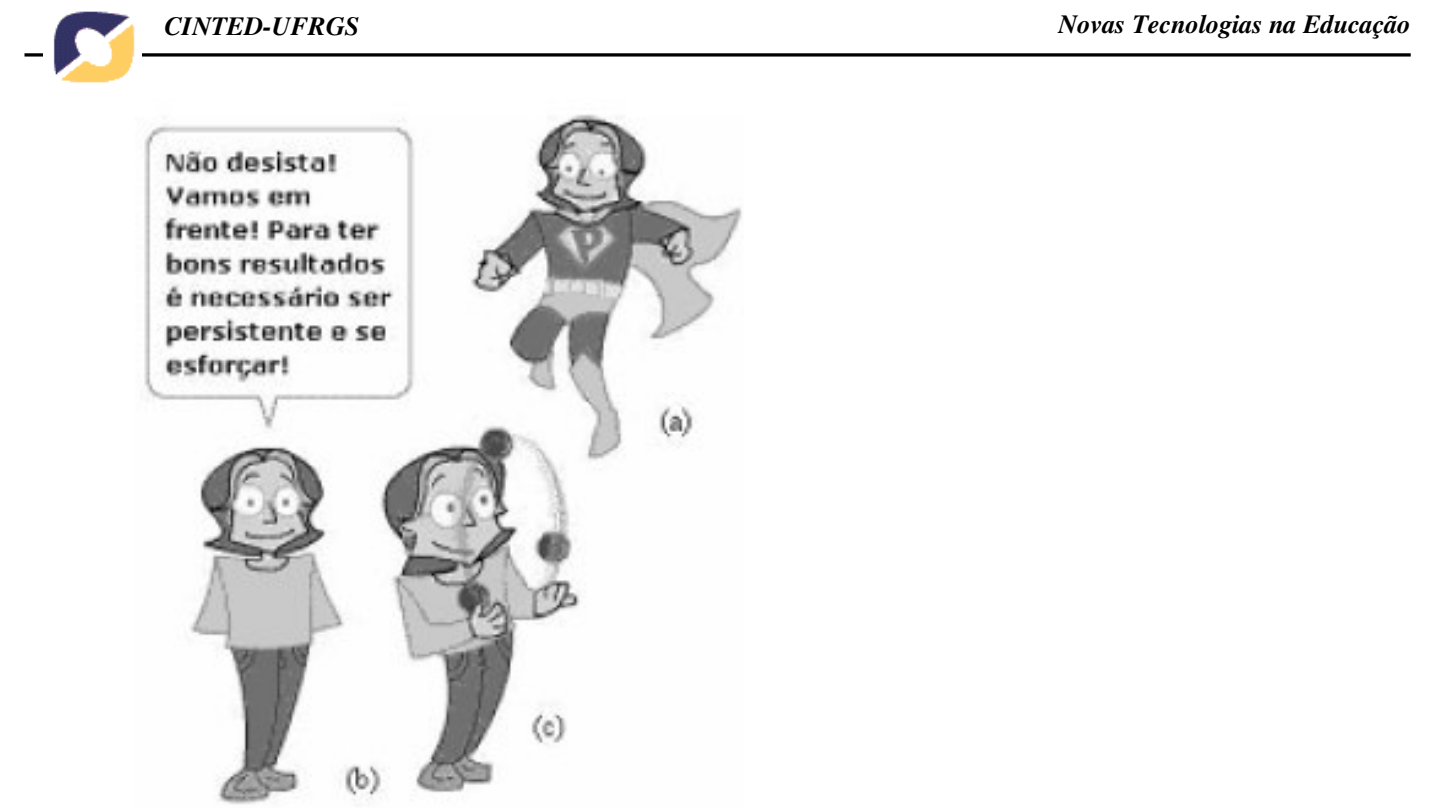

Figura 4 - Comportamentos da PAT

\section{InteliWeb - Projeto Piloto}

O Inteliweb é um ambiente de aprendizagem na WEB desenvolvido na linguagem Java, utilizando Servlet e JSP (Murach 2003). E é neste ambiente que os agentes AGMA e PAT serão acoplados.

O material didático disponibilizado pelo ambiente sobre o conteúdo "Flor" foi elaborado pelo professor da disciplina Anatomia Vegetal, do curso de Biociências da UFRGS juntamente com a da psicóloga instrucional do projeto, os quais procuraram desenvolver um material interativo para intensificar a compreensão, absorção e domínio do assunto pelo aluno.

A interatividade entre o material e o aluno é possibilitada mediante o uso de suportes audiovisuais e hipermídia interativa e, entre o aluno e professor, mediante os meios de comunicação disponíveis (e-mail e encontros presenciais, visto que a disciplina é presencial). O ambiente disponibiliza o conteúdo dividido em tópicos, os quais são apresentados em três diferentes formas de apresentação (HTML com figuras, animações FLASH e Vídeos explicativos).

Após as telas de apresentação do ambiente desenvolvido e de identificação e autenticação do aluno, o mesmo pode interagir com a interface do Ambiente, como mostra a Figura 5, a qual é composta por três partes, são elas: Pastas (Início, e-mail, Conteúdo); Barra de Ferramentas com várias funcionalidades disponibilizadas e um Espaço Visual

(visualização do material didático ou demais opções da Ferramenta). 


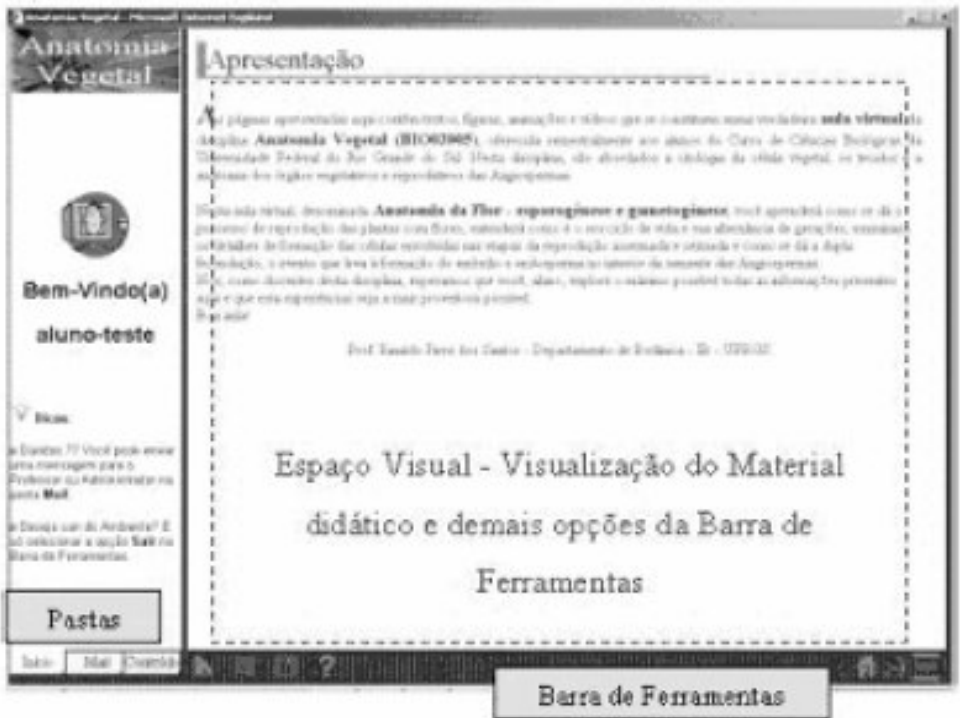

Figura 5 - Interface do InteliWeb

A pasta Início apresenta uma mensagem de Boas Vindas, Dicas e eventuais mensagens da Ferramenta ao aluno, como por exemplo: "Mensagem enviada com sucesso.", na pasta Mail é possível enviar mensagens para usuários cadastrados (aluno, professor ou administrador) e a pasta Conteúdo apresentam o sumário do curso a ser acessado pelo aluno na sessão de estudo.

A Barra de Ferramentas apresenta sete opções, são elas (da esquerda para a direita): Download (material didático disponível para download), Glossário (apresenta um vocabulário das palavras citadas no material didático) o material didático possui referências (links) para o glossário, Ler Mensagem (opção de leitura/exclusão/resposta a mensagens recebidas), Help (ajuda do Sistema - informações de como utilizar as opções disponíveis na ferramenta), Formas de Apresentação (cada vez que é escolhido um material didático o ambiente requer a escolha de uma das três formas de apresentação deste material - o material pode ser visualizado várias vezes e em qualquer forma que o aluno selecionar), Pessoal (troca de senha e dados pessoais do aluno, como por exemplo, rendimento no material e tempo na sessão) e Sair (finaliza a sessão e fecha a janela).

\subsection{Procedimento de Avaliação do Ambiente Piloto}

A avaliação do ambiente ocorreu no semestre 2004/1 sem a presença dos agentes. A turma foi divida em duas, sendo que 25 alunos tiveram aula presencial (expositiva com o professor) e os demais 25 alunos se propuseram a participar do experimento. Todos os alunos responderam o questionário "Nível de Competência Tecnológica" elaborado por (COSTA, 2002), o qual detecta o nível em que se encontra o aluno no que diz respeito à utilização do computador. Os resultados obtidos foram que $42 \%$ dos alunos que se submeteram à aula virtual são principiantes no uso do computador, enquanto que $58 \%$ têm experiência assinalável na utilização do computador.

Os alunos que participaram do experimento responderam um pós-questionário para captar as suas percepções quanto ao material didático, incluindo os recursos didáticos (teoria e exercícios) e formas de apresentação para a teoria (HTML, FLASH e VÍDEO), exercício (múltipla escolha e cruzadas) e facilidade ao usar a interface. Como resultados obteve-se uma percepção positiva quanto ao material teórico e exercícios, como por exemplo, os alunos consideraram a interface agradável e fácil de utilizar. 


\section{Conclusões}

Em ambientes de ensino é fundamental que se oriente o aluno a estudar com autonomia, desenvolvendo habilidades de independência e iniciativa. E, além disso, a combinação da captura das crenças de auto-eficácia com a utilização de um agente pedagógico animado propiciarão o aumento da interação entre alunos e computadores, e incrementa a habilidade do computador para engajar e motivar o aluno.

A modelagem do comportamento cognitivo do aluno apresenta uma grande quantidade de incertezas e ruídos e o processo de inferência é incompleto e pode ser baseado em conhecimento inconsistente. Estes fatores levaram a seleção da Lógica Fuzzy para mapear as crenças da auto-eficácia que compõem a principal característica do modelo de aluno proposto neste trabalho.

A utilização da característica individual da auto-eficácia, do Modelo Motivacional de Bandura (1997), é importante para a modelagem cognitiva do aluno, na medida em que, conforme Bandura, é ela que permeia o controle e regulação pessoal do aluno na sua motivação durante a construção do seu conhecimento no processo de aprendizagem.

Nesse sentido, quanto mais um sistema computacional (tutor) tiver a capacidade de capturar a auto-eficácia do aluno, tanto mais este poderá fomentar no aluno a crença na sua própria capacidade de exercer controle sobre a sua aprendizagem.

O próximo experimento será realizado com os agentes integrados ao InteliWeb. Deste trabalho resultarão um estudo comparativo entre a utilização do InteliWeb com e sem os agentes, um estudo da adequação da Lógica Fuzzy como ferramenta para inferir a autoeficácia do aluno e estudo da adequação do recurso de feedbacks através de um agente pedagógico animado com comportamentos especialmente selecionados para aumentar a auto-eficácia e motivação do aluno.

\section{Agradecimentos}

Este projeto é financiado pelo CNPq através de bolsas de doutorado. 


\section{Referências bibliográficas} (1997)

BANDURA, A. Self- Efficacy - The exercise of control. New York: Freeman.

BERCHT, Magda. Em direção a agentes pedagógicos com dimensões afetivas. 2001. 152 p. : il. Tese (doutorado)-Universidade Federal do Rio Grande do Sul. Instituto de Informática. Programa de Pós-Graduação em Computação. Porto Alegre, BR-RS(2001).

BZUNECK, Boruchovitch. A Motivação do Aluno: Contribuições da Psicologia Contemporânea. Petrópolis: Editora Vozes; 116-133. (2004).

CERDEIRA, José Pedro. A percepção da eficácia pessoal e os mecanismos de autoregulação das aprendizagens. In Revista Portuguesa de Pedagogia, Ano XXIX, n. 2, p.137- 159. (1995)

COSTA, F. A. Elementos para reflexão sobre a integração das TIC na Educação, in Lourdes Marcelino Machado, Naura Syria Carapeto Ferreira (orgs.) Política e gestão da educação - dois olhares, S.Paulo, DP\&A Editora. (2002)

JAQUES, P et al. Cognitive Reasoning to Respond Affectively to the Student. In: The IASTED International Conference on Computers and Advanced Technology in Education. Proceeedings... Rhodes, Greece.(2003)

JOO, Y., BONG, M., \& CHOI, H.. Self-efficacy for self-regulated learning, academic selfefficacy, and Internet self-efficacy in Web-based instruction. Educational Technology Research and Development, 48(2), 5-17. (2000)

JOHNSON, D. W. \& JOHNSON, F. P.. Joining Together: Group Theory and Group Skills. Boston, MA: Allyn and Bacon. (1997)

KAY, J.. Learner control. User Modelling and User Adapted Interaction 11(1-4), 111-127. (2001)

LESSER, V. et al. Distributed Sensor Networks: A Multiagent Perspective. Kluwer Academic Publishers.(2003)

LEVINE, T. et al. Computer use, confidence, attitudes, and knowledge: A causal analysis. Computers in Human Behavior, 14(1), 125-46. Journal of Personality and Social Psychology, 815-822.(1998)

LOCKE, E.A. A Theory of goal setting and task performance. Englewood Cliffs. NJ: Prentice Hall. (1990)

PAPI, Disponível em: < http://edutool.com/papi>. Acesso em 11 mar. 2005..

PETERS, O. Didática do Ensino a Distância. Experiências e estágio da discussão numa visão internacional. S. Leopoldo, Editora Unisinos. (2001)

MADDUX, J. Self-efficacy theory: An introduction. In J. Maddux (Ed.) Selfefficacy, adaptation and adjustment. New York: Plenum Press. (1995)

O'MALLEY, J.et al.. Student's perception of distance learning, online learning and the traditional classroom. Online journal of distance learning administration, 2(4). (1999)

SILVEIRA, R.et al. JADE: java agents for distance education framework: an agent oriented modeling approach for distributed intelligent learning environment projects. Em: International Conference of Information and Communication Technologies in Education. Proceedings. (2002)

SOLDATO, T.; BOULAY,B. Implementation of Motivational Tactics in Tutoring Systems. Journal of Artificial Intelligence in Education, Charlottesville, v.6, p.337-378, (1995).

TEDESCO, P. Using group models to support group planning interactions in MarCo. In Anais do XII Simpósio Brasileiro de Informática na Educação, pp. 202-210. Vitória: Sociedade Brasileira de Computação. (2001)

WOOLDRIDGE, M. J et al. Reasoning about Rational Agents. The MIT Press (Cambridge, Massachussetts/London, England). 2000. 